\title{
Low Energy Emulsification Methods for Nanoparticles Synthesis
}

\author{
Veronique Sadtler ${ }^{1}$, Johanna M. Galindo-Alvarez ${ }^{1}$ \\ and Emmanuelle Marie -Bégué ${ }^{2}$ \\ ${ }^{1}$ Laboratoire Réactions et Génie des Procédés - GEMICO, \\ CNRS-Nancy Université, Nancy \\ ${ }^{2}$ UMR 8640 CNRS-ENS-UPMC, Ecole Normale Supérieure, \\ Paris, \\ France
}

\section{Introduction}

Nanoparticles synthesis by miniemulsion polymerization produces materials that are not obtainable by means of other techniques such as conventional emulsion polymerization. The reason is that, in miniemulsion polymerization, particles are mainly formed by droplet nucleation (Asua, 2002). However, the high energy requirement for preparation of nanoemulsions by traditional methods (Mason et al., 2006; Solans et al., 2005) has precluded widespread use and commercialization.

Nanoemulsions, also referred as miniemulsions or ultrafine emulsions, compose a particular class of emulsions consisting of colloidal dispersions, transparent or bluish for the smallest droplet sizes between $20-100 \mathrm{~nm}$, or milky for sizes up to $500 \mathrm{~nm}$ (Solans et al., 2002). In opposition to microemulsions, these systems are thermodynamically unstable, and the droplet size tends to increase with time before phase separation. Nevertheless, the very small initial droplet size makes them kinetically stable (Tadros et al., 2004).

As nanoemulsions are non-equilibrated systems, external energy is required for their preparation. Two generating processes are reported in the literature. In the first case, high mechanical energy is applied during emulsification, generally by using high shear stirring, high pressure homogenizers and/or ultrasound generators. On the contrary, the lower energy method, or condensation method, is based on the phase transitions taking place during the emulsification process (Lamaallam et al., 2005; Solans, et al., 2002; Tadros, et al., 2004). These phase transitions result from changes in the spontaneous curvature of the surfactant and can be achieved (i) at constant composition by changing the spontaneous curvature of non-ionic surfactants with temperature, the well-known Phase Inversion Temperature, PIT, widely used in industry (Izquierdo et al., 2005; K. Shinoda \& Saito, 1968)or (ii) at constant temperature by varying the composition of the system by the Emulsion Inversion Point (EIP) method (Forgiarini et al., 2001; Pey et al., 2006; Porras et al., 2008). 
Thus, nanoemulsions are specially formulated heterophase systems where stable nanodroplets (with a diameter lower than $500 \mathrm{~nm}$ ) of one monomer phase are dispersed in a second continuous phase before polymerization takes place, often following a radical mechanism. Since its introduction, this approach has extended the classical emulsion radical polymerization, as ideally each nanodroplet could be regarded as an individual batch reactor, a nanoreactor. Indeed, when (oligo) radicals are generated in the continuous phase, nanodroplets compete with micelles for their capture. In addition, the amount of surfactant added in the feed is usually adjusted so as to minimize (or avoid) the presence of micelles in the continuous phase (Anton et al., 2008; Antonietti \& Landfester, 2002).

In those conditions, it could be a good approximation to consider that each droplet behaves as an independent reaction vessel, a hypothetical bulk state where the continuous phase may still transport initiators, side products and heat. Thus, miniemulsion polymerization allows preparing water-based formulated polymers with high solid contents. Additionally this particular mode of design of nanoparticles becomes an advantage, since the chemical composition and colloidal characteristics of the initial nanoemulsion can be used to prepare polymer nanoparticles by "miniemulsion polymerization" of the monomer contained in the oil droplets. The nanoemulsions used for that purpose are mainly prepared by high-energy emulsification methods (Asua, 2002). The aim of this chapter is to show the EIP Method and the Near - PIT concept as a tool to produce miniemulsion templates for miniemulsion polymerization.

\section{Nanoparticles by Emulsion Inversion Point (EIP) method}

Studies showed that nanoemulsions with very small droplet sizes can be obtained through low-energy methods if, during the emulsification process, the oil is completely dissolved in a single phase, like a bicontinuous microemulsion or a lamellar crystalline phase (Mohlin et al., 2003; Rang \& Miller, 1999).The further evolution of the system led to the dislocation of this continuous phase into small nanodroplets. For instance, in the EIP method, the addition of water to a system of water/oil/surfactant forming a lamellar phase increases the hydratation degree of the surfactant polar head thereby increasing its spontaneous curvature. The lamellar phase is disrupted, and the oil, which was initially dissolved, forms small droplets in the size order of the thickness of the hydrophobic layer (See Figure 1). Such methods of nanoemulsion preparation have received increasing attention (Maestro et al., 2008), since even active molecule (i.e. lidocaïne) encapsulation in emulsions is achievable by these protocols (Sadurní et al., 2005).

As already mentioned, nanoemulsions can be used to prepare polymer nanoparticles by miniemulsion polymerization of the monomer contained in the oil droplets. The nanoemulsions used for that purpose are mainly prepared by sonifiers and high-pressure homogenizers (Asua, 2002). Only a few studies described the preparation of nanoparticles from nanoemulsions obtained by condensation methods (Calderó et al., 2011; Isabel Solè et al., 2010; Liat Spernath \& Magdassi, 2007; L. Spernath et al., 2009).

In this section the formation by EIP, of monomer-in-water nanoemulsions, followed by their conversion in polymer nanoparticles will be considered (Sadtler et al., 2010). For this purpose, the water/Brij 98/styrene system was chosen. Brij compounds are POE-based non ionic surfactants which are commonly used for biomedical applications. Water always 


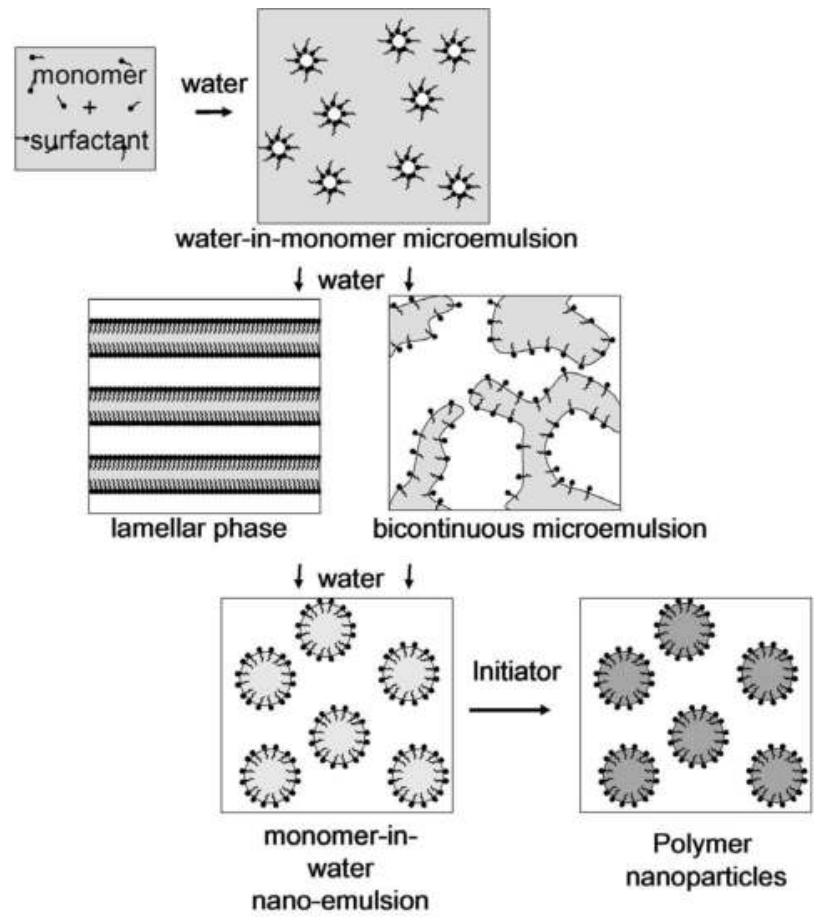

Fig. 1. Schematic representation of the polymer nanoparticles synthesis from nanoemulsions prepared by the EIP method (Adapted from Sadtler, et al., 2010)

contained $0.1 \mathrm{M}$ of $\mathrm{NaCl}$ and a small quantity of hexadecane was always solubilized in styrene (in the 5:95 ratio) to avoid Ostwald ripening after nanoemulsion formation (Kabal'nov et al., 1987; Marie et al., 2007). Because lamellar liquid crystalline phase and/or bicontinuous microemulsions are necessary to generate nanoemulsions, the partial phase diagram of the system was determined prior to the nanoemulsion preparation and its miniemulsion polymerization. The phase diagram has been carried out at $50^{\circ} \mathrm{C}$ due to the suitable temperature to styrene polymerization (Figure 2).

The phase behaviour was found to be in good agreement with the one of other systems containing polyoxyethylene alkyl ether non-ionic surfactant of technical grade. A domain of liquid isotropic phase extends along the surfactant/styrene axis solubilizing up to $10 \%$ water. According to the literature, the most probable structures are inverse micelles or $\mathrm{W} / \mathrm{O}$ microemulsion (Om). Higher amounts of water (up to $20 \%$ approximately) led to the appearance of the lamellar crystalline phase $(\mathrm{L} \alpha)$ that coexists with the Om phase. A wide multiphasic region, with two or three phases comprising liquid crystalline phases (equilibrium not determined), occupies the centre of the diagram for water composition from $20 \%$ to $60 \%$. The lamellar crystalline phase in equilibrium with water, $(W+L \alpha)$ is observed at high-medium surfactant concentration. For the higher amounts of water (up to $90 \%)$, the two phase region is present: oil-in-water microemulsion $(\mathrm{Wm})$ and free oil $(\mathrm{O})$. Above this area, by increasing the surfactant concentration, the oil is completely 
incorporated in the oil-in-water microemulsion. Finally, at low Brij 98 concentrations (O:S ratio above 80:20), appears the three-phase region (Forgiarini, et al., 2001; I. Solè et al., 2006).

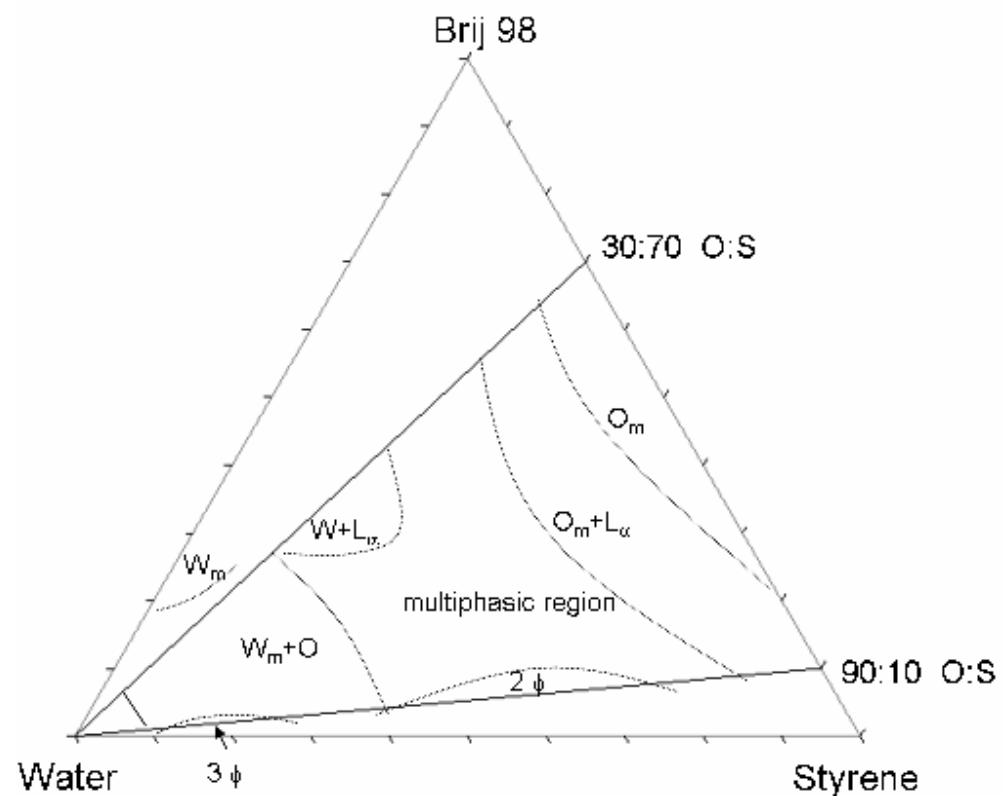

Fig. 2. Partial phase diagram of the water/Brij $98 /$ styrene system, at $50^{\circ} \mathrm{C}$. Wm: direct micellar solution or $\mathrm{O} / \mathrm{W}$ microemulsion; Om: reverse micellar solution or $\mathrm{W} / \mathrm{O}$ microemulsion); L $\alpha$ : anisotropic phase (lamellar liquid crystalline phase); W: water phase; O: oil phase. $2 \phi$ : two isotropic liquid phases; $3 \phi$ : three isotropic liquid phases. (Adapted from Sadtler, et al., 2010)

The equilibrium phase diagram (figure 2), allows identifying a suitable region for nanoemulsion formation. This domain corresponds to the two-phase region, $\mathrm{Wm}+\mathrm{O}$, for $\mathrm{O} / \mathrm{S}$ ratio between $30: 70$ and $80: 20$. The emulsification process path is schematically represented by an arrow on the phase diagram (figure 3). Thus, the addition of water at constant rate to different mixtures of Brij 98 and styrene (inside the suitable region), allowed the system to cross the multiphasic central region, with two or three phases comprising liquid crystalline phases, favouring nanoemulsion formation. The final water concentration was fixed at $80 \mathrm{wt}$. \%, to keep a relatively high percentage of dispersed phase. These aqueous dispersions can be regarded as $\mathrm{O} / \mathrm{W}$ nanoemulsions (and not microemulsions) because they are formed in the multiphase region $\left(\mathrm{W}_{\mathrm{m}}+\mathrm{O}\right)$. Bluish dispersions were obtained. After addition of KPS solution (the water soluble thermal initiator), miniemulsion polymerization was carried out at $50^{\circ} \mathrm{C}$ for $24 \mathrm{~h}$.

Figure 4 shows the evolution of nanoparticle sizes as a function of O:S ratio for nanoemulsion containing 80 wt.\% water. Results from Sadtler et al., (2010), showed that nanoparticle sizes were clearly dependent on O:S ratio and increased with O:S ratio. After polymerization, nanoparticle sizes varied between $36 \mathrm{~nm}$ (O:S ratio of around 0.5) and 


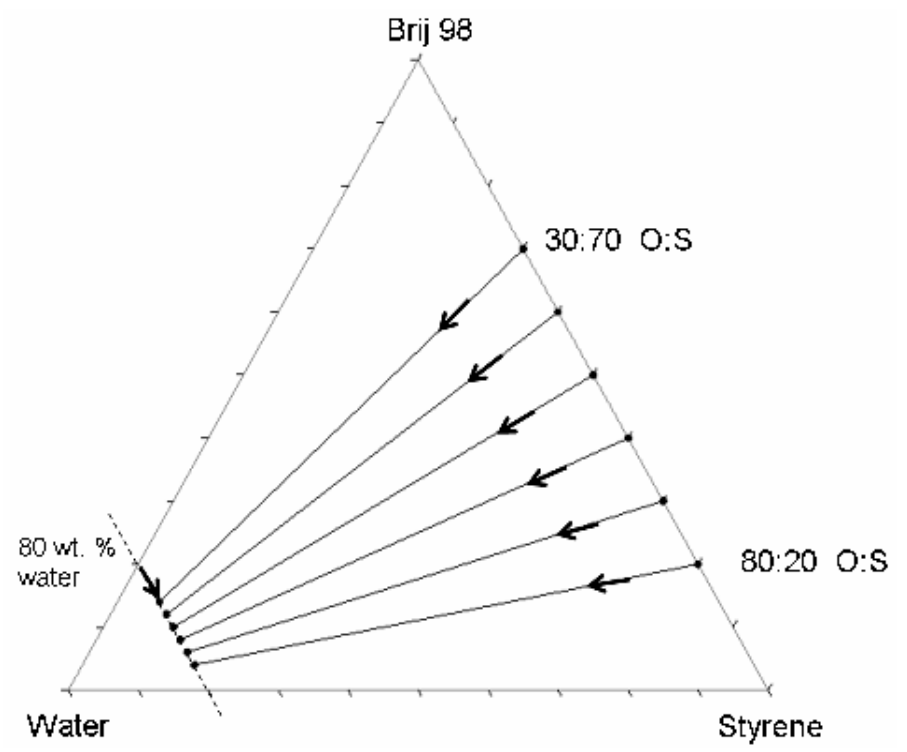

Fig. 3. Schematic representation of the emulsification paths: stepwise addition of $80 \mathrm{wt}$. \% water to different oil:surfactant ratios mixtures

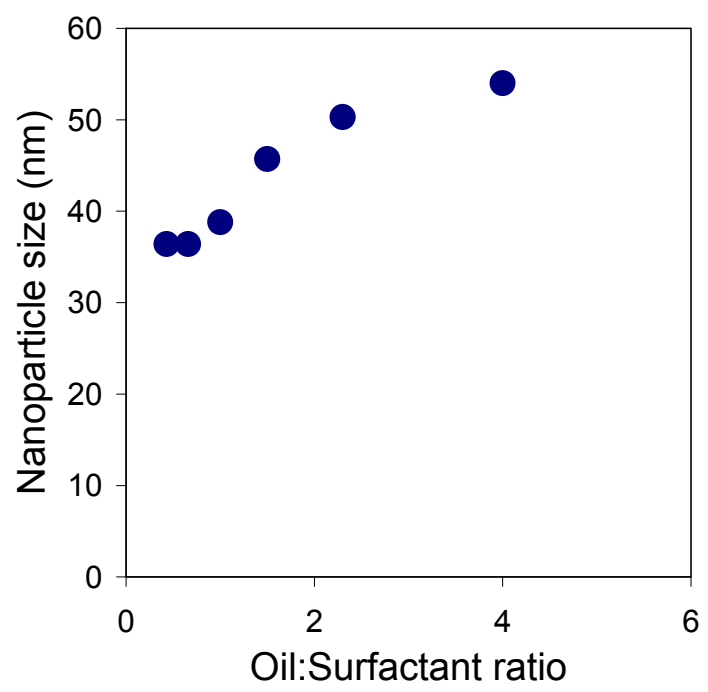

Fig. 4. Polystyrene nanoparticle diameter as function of O:S initial ratio (water addition rate $=4.6 \mathrm{ml} / \mathrm{h}$ ). (Adapted from Sadtler, et al., 2010)

$50 \mathrm{~nm}(\mathrm{O}: \mathrm{S}$ ratio $=4)$. The fact that the nanoparticle diameter progressively increased with the oil:surfactant ratio suggests that the styrene constituted the inner core of the nanodroplets, which was consistent with a direct $\mathrm{O} / \mathrm{W}$-type structure. It should be noticed that the polystyrene nanoparticles sizes obtained by this emulsification path, were 
exceptionally small for the water/Brij 98/styrene system, compared to those reported in the literature from high-energy emulsification method (Antonietti \& Landfester, 2002; Asua, 2002; Bouanani et al., 2008; Marie, et al., 2007).

Figure 5 shows the size of the nanoparticles synthesized from nanoemulsions prepared at different water flow rates, ranging from $4 \mathrm{ml} / \mathrm{h}$ to $150 \mathrm{ml} / \mathrm{h}$, for a O:S ratio of 30:70 and final water composition of $80 \mathrm{wt}$. \%. As predicted by Pey (2006), the polystyrene particle sizes increased with water addition rate. This could be related by the crossing rate of the phases along the emulsification paths, i.e. the kinetic of the whole emulsification process.

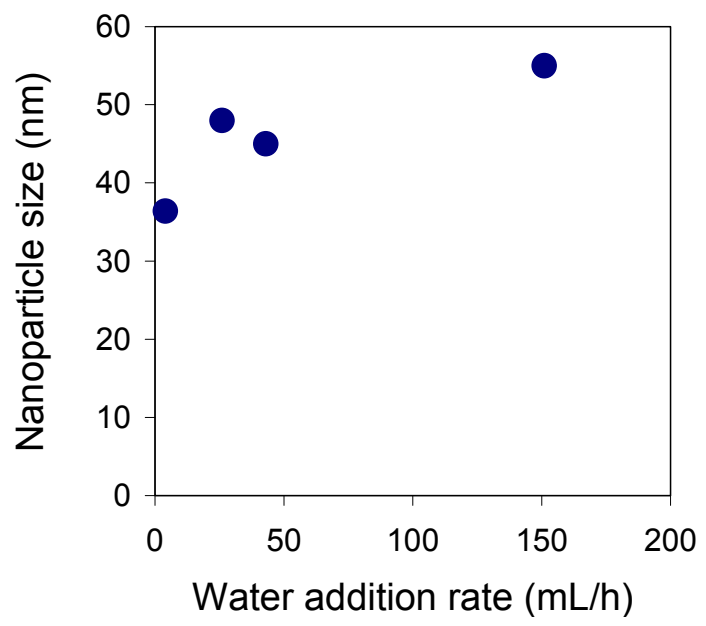

Fig. 5. Polystyrene nanoparticle size as function of the water addition rate $(\mathrm{O}: \mathrm{S}$ ratio $=0.42)$. (Adapted from Sadtler, et al., 2010)

To confirm this point, the same path was followed as in the first experiment by adding water at once. In the second experiment, the order of the addition was modified: the oil was added at once to the mixture of water and surfactant (the sample compositions were identical in the two cases).

Figure 6 presents the particle sizes after polymerization obtained following these different pathways. Particles resulting from stepwise addition of water over the mixture of oil and surfactant were smaller than the one obtained by water addition at once (36 and $65 \mathrm{~nm}$ respectively).

Phase transitions that take place during the emulsification process (as result of the change in the spontaneous curvature of surfactant), allow to low energy emulsification methods make use of stored chemical energy to get a small drop size distribution. However, when styrene was added to the water and Brij 98 mixture (at once), milky emulsions were formed and the polymerization of the oil droplets did not produced small nanoparticles. Hence the resulting polystyrene dispersion presented an average size of $420 \mathrm{~nm}$ (figure 6). The polymerization process might even be totally different in this case, switching from real miniemulsion polymerization to a "simple" emulsion polymerization process. Indeed, miniemulsion polymerization is only possible if the droplets are nucleated thereby leading to 
polymerization inside the droplets. The smaller the droplet size, the higher the probability of radical entry into the droplets because of the higher interface area. When the emulsion droplets are bigger, the radical entry probability decreases while the micellar and/or homogeneous nucleation increased. Theses nucleation processes are found in the emulsion polymerization process.

Concerning the emulsification protocol, internal phase addition should favour a proper mixing, to assure to reach the equilibrium with all the oil dissolved into the critical phases (i.e., cubic liquid crystal or lamellar phase) (Isabel Solè, et al., 2010).

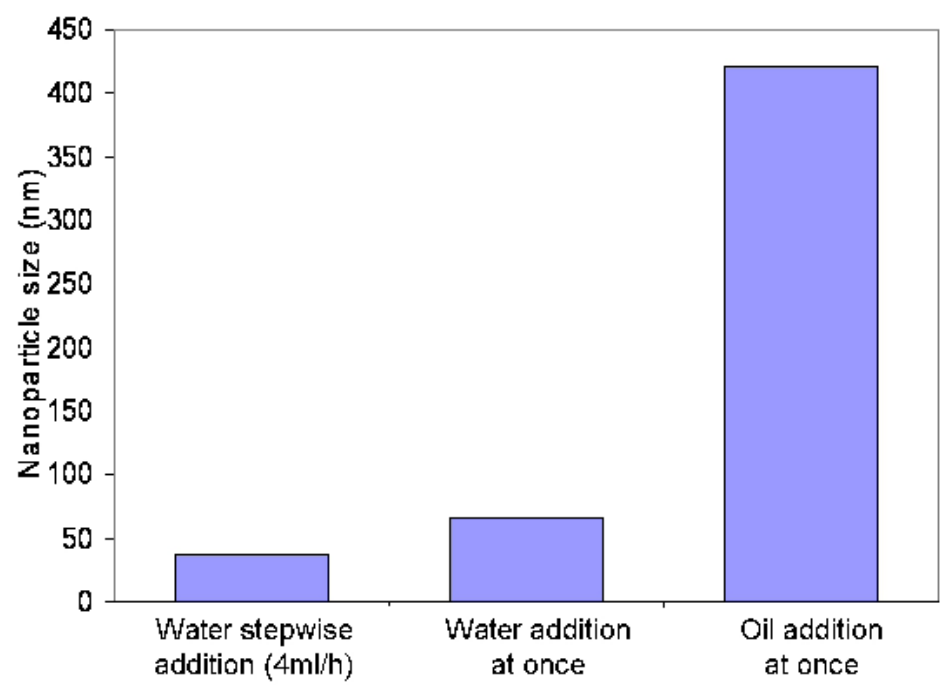

Fig. 6. Polystyrene nanoparticle diameter as function of the emulsification process. The nanoemulsions were prepared at 30:70 O:S ratio, with a final water concentration of $20 \mathrm{wt} . \%$.

\section{Nanoparticles by near-PIT method}

The formulation-composition map is the graphical representation of the so-called generalized formulation (see Figure 7). The middle shaded zone corresponds to the three phase behavior at or near the optimum formulation. The formulation variable scale is such that the hydrophilicity increases from top to bottom and the stair like bold line is the standard inversion frontier. This line separates the regions in which $\mathrm{O} / \mathrm{W}$ and $\mathrm{W} / \mathrm{O}$ emulsions are formed as the result of the stirring of an equilibrate surfactant-oil-water system (Salager, 2000b; Salager et al., 1983). The crossing over through the inversion frontier represents a dynamic phase inversion, since the curvature of the liquid-liquid interface swaps its bending from one way to the other. This change is the consequence of the variation in one of formulation variables (i.e., surfactant affinity) or composition variables (i.e., oil/water ratio) during the stirring process.

If the change is rendered in the map as a vertical shift (crossing through the horizontal branch of inversion line), as for instance in the continuous change in temperature (in the case of non-ionic surfactant), the inversion will always take place under the same conditions 
(at so-called optimum formulation). Such a dynamic inversion, which is found to be reversible, has been called transitional because it is linked to a phase behavior transition (Salager, 2000a). On the other hand, when the inversion takes place by crossing through a vertical branch of the inversion line, it is called catastrophic because it may be modeled by using catastrophe theory (Salager, 1988; Salager, et al., 1983).

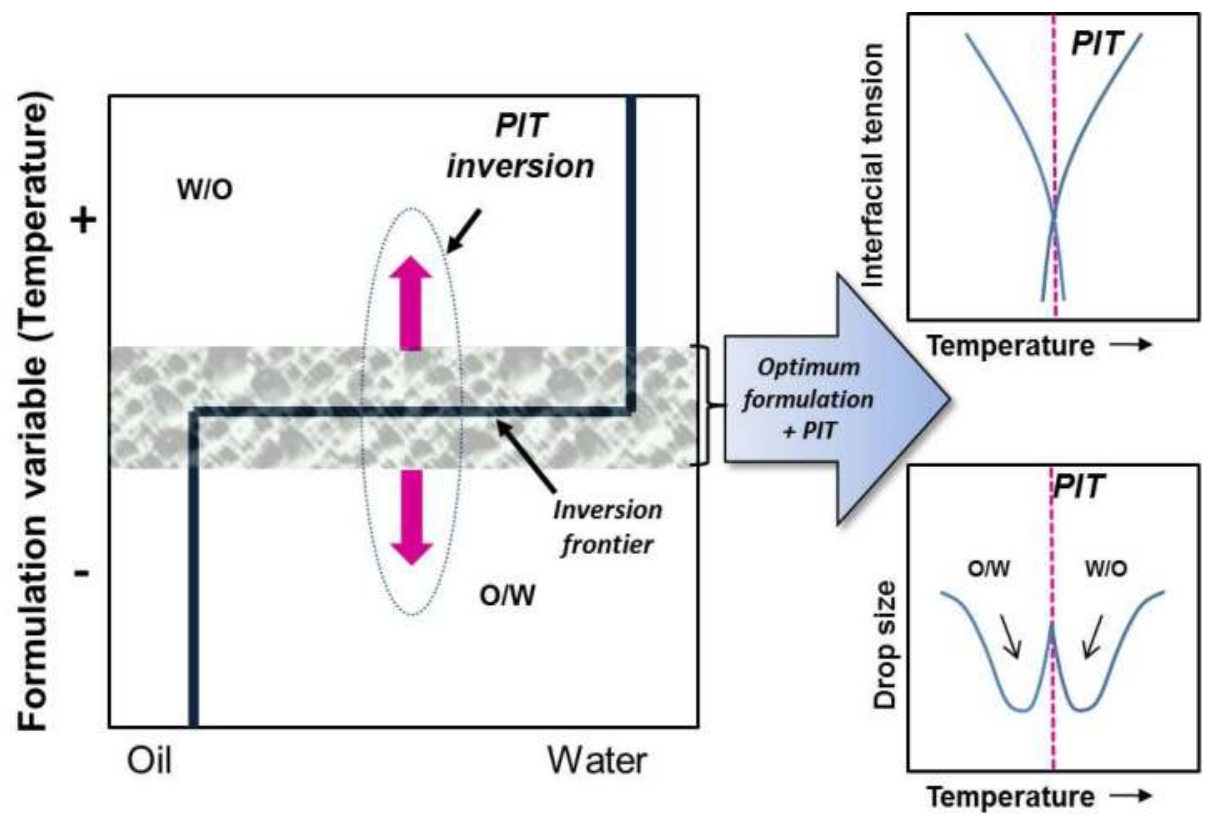

Fig. 7. Formulation - composition map. The bold line is the standard inversion frontier. At right side upper schema illustrates the minimum of interfacial tension obtained near to PIT value. Bottom schema show the regions near to PIT value where droplet size presents a minimum.

The transitional phase inversion is based on the particular ability of emulsions stabilized by poly(ethylene oxide) (PEO)-based non-ionic surfactants to undergo a phase inversion upon temperature variation (Kõzõ Shinoda \& Arai, 1964). A change of formulation (i.e. induced by temperature increase) along a vertical line, results in a minimum of both the interfacial tension and the emulsion stability at the optimum formulation (see Figure 5). The minimum of stability at optimum formulation has been attributed either to the percolation through liquid crystals located across the thin film, or to the trapping of all surfactant in the microemulsion (Antón et al., 1986). Thus as optimum formulation is approached (either from above or from below the standard inversion line) both the interfacial tension and the emulsion stability decrease. As far as the emulsion droplet size is concerned, the two resulting effects are opposite. The weakening of interfacial tension tends to enhance the efficiency of stirring-mixing process and thus produces smaller droplets, while the decrease in emulsion stability favors the occurrence of coalescence events, and thus results in larger droplets (Salager et al., 1996) (see Figure 7). 
The use of the low-energy PIT method has been reported for miniemulsion polymerization by heating above PIT temperature to inverse the emulsion and then cooling to induce the reinversion followed sometimes by a rapid cooling in an ice bath to set droplet size within the submicronic range (Jahanzad et al., 2007; Liat Spernath \& Magdassi, 2007; L. Spernath \& Magdassi, 2010). In this section the Near - PIT method (Galindo-Alvarez et al., 2011), for which the strong decrease in interfacial tension near to optimum formulation is used to form submicronic droplets, will be discussed. In contrast to other protocols, Near-PIT method does not reach and cross temperatures aboves PIT, thus temperature sensitive molecules can be use through a carefully match of surfactant system.

It has been reported that stable $\mathrm{O} / \mathrm{W}$ nanoemulsions can be produced by the PIT method if the dispersed system is rapidly cooled by about $30^{\circ} \mathrm{C}$ away from its temperature of transitional phase inversion (Solans, et al., 2005). In those conditions, droplet coalescence becomes negligible because the non-ionic surfactant molecules provide an efficient steric barrier. Therefore the miniemulsion templates should exhibit a PIT value about $30^{\circ} \mathrm{C}$ higher than the targeted polymerization temperature. PIT value results from interaction between overall surfactant concentration, surfactant mixing ratio and weight fraction oil (K. Shinoda \& Arai, 1967). Thus, the PIT value of the studied system was tuned by the appropriate selection of the constituents.

In the case of non-ionic surfactant mixtures, it is well-known that increasing the length of the poly(ethylene oxide) chain results in higher HLB numbers and thus the increase in PIT. Two non-ionic surfactants, PEO stearyl ethers (Brij 78 and Brij 700), differing by the length of the PEO chain (20 and 100 repeat units, respectively) were used for formulating the nanoemulsions allowing a certain adjustment of the PIT value within the convenient range. Figure 8 shows the influence of surfactant mixing ratio and weight fraction of dispersed phase over PIT value. Thus a water/Brij $78+$ Brij $700 /$ styrene system containing $1 \% \mathrm{w} / \mathrm{v}$ of $\mathrm{NaCl}$, a surfactant mixing ratio of $0.35 / 0.65$ Brij $700 /$ Brij 78 and 35 wt $\%$ of dispersed phase with PIT value around $80^{\circ} \mathrm{C}$ has been chosen to carry out the miniemulsion polymerization at $50^{\circ} \mathrm{C}$ using potassium persulfate (KPS) as water-soluble initiator.

On the basis of the previously selected formulation, the Near-PIT emulsification procedure is designed and compared to classical sonification and emulsion polymerization with regard to the final nanoparticle size obtained after reaction completion. For used conditions, a polymerization temperature of $50{ }^{\circ} \mathrm{C}$ ensures fast enough initiator decomposition so that no limitation by the polymerization reaction is considered. About particle nucleation mechanism, droplet size distribution of miniemulsion polymerization templates was similar to droplet size distribution of latex particles, suggesting predominance of droplet nucleation mechanism.

Near-PIT protocol, as discussed at the beginning of this section, is based on the effect that droplet size decreases when PIT temperature is approached as the result of an enhanced stirring efficiency due to the very low interfacial tension. Nevertheless, in that temperature range, close to the PIT, resulting emulsions turn out to be very unstable; and no theoretical relationships are available to discriminate zones of minimum droplet size from unstable emulsion. In a general way, in Near-PIT protocol the system is heated until a temperature close to PIT value, equivalent to: $-5^{\circ} \mathrm{C}$ or $-10^{\circ} \mathrm{C}$ below PIT temperature. As 
this value is particular for each system formulation, Figure 9 illustrates the final average particle diameters obtained for suspensions resulting from miniemulsion polymerizations after Near-PIT protocols carried out at PIT $-10^{\circ} \mathrm{C}$, PIT $-5^{\circ} \mathrm{C}$ and PIT- $0.5 \circ \mathrm{C}$ values. The used formulation was $\phi_{\mathrm{oil}}=0.2, \mathrm{X}_{\mathrm{Brij700}}=0.35$ and the overall surfactant concentration equal to $5.2 \mathrm{wt} \%$. For the three examined conditions, particles with diameters lower than $100 \mathrm{~nm}$ were obtained after polymerization. In addition, polydispersity indices were relatively low $(<0.22)$ indicating reasonably narrow size distributions. Even if the average diameters were similar for the different temperatures, the better compromise was obtained for the experience carried out at PIT $-5^{\circ} \mathrm{C}$, since its polydispersity index was the lowest.

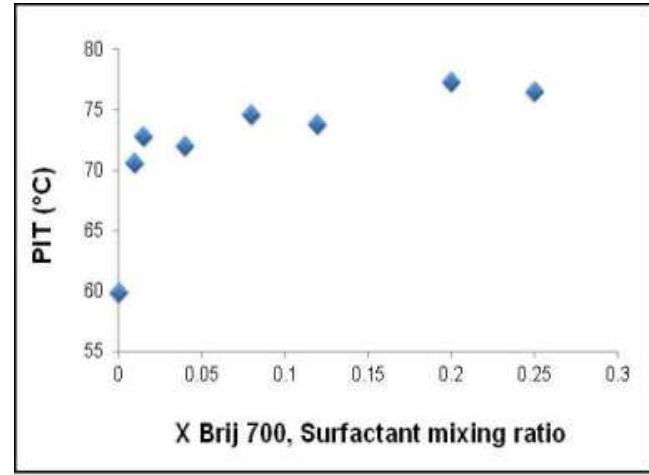

a)

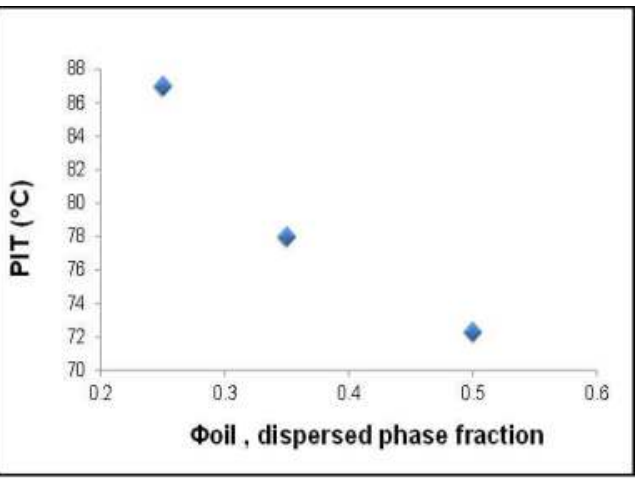

b)

Fig. 8. a) Increase of PIT value as function of Brij ${ }^{\circledR} 700$ content, $X B$ rij700, $\phi_{\text {oil }}=0.5$ and $8 w t \%$ of surfactant concentration. b) Variation of PIT value with the weight fraction of dispersed phase, $\phi_{\mathrm{oil}}, \mathrm{X}_{\mathrm{Brij} 700}=0.25$ and $8 \mathrm{wt} \%$ of surfactant mixture.

(Adapted from Galindo-Alvarez, et al., 2011)

In miniemulsion polymerization, the use of an effective surfactant system may give very small (20-300 $\mathrm{nm})$ monomer droplets with very large surface area and almost all the surfactant adsorbed at the droplet surface (the concept of critically "stabilized miniemulsion"). Particle nucleation occurs primarily via radical (primary or oligomeric) entry into monomer droplets, since little or no surfactant is present in the form of micelles. The reaction proceeds by polymerization of the monomer in these small droplets, since the loci of polymerization become the monomer droplets and ends when all monomer in droplet is consumed (Schork et al., 2005).

In contrast for macroemulsion polymerization, polymerization starts with large monomer droplets (diameters higher than $10 \mu \mathrm{m}$ ) stabilized by surfactant and coexisting with empty or monomer-swollen surfactant micelles. The water-soluble initiator forms oligoradicals 
with the slightly water-soluble monomer molecules and these oligoradicals go inside the micelles (heterogeneous nucleation) or start nucleate particles in the continuous phase after reaching a critical degree of polymerization (homogeneous nucleation). During polymerization, the monomer diffuses from the large monomer droplets through the continuous phase to the polymer particles and sustain polymer particle growth until the monomer droplets have vanished (Antonietti \& Landfester, 2002). Thus in miniemulsion polymerization latex particles size distribution are expected to correspond to the primary emulsion droplets. On the contrary, in macroemulsion polymerization the particle size distribution is established by the contribution of several nucleation processes leading to average diameters usually larger than $100 \mathrm{~nm}$ and sometimes to the formation of several populations within the final sample.

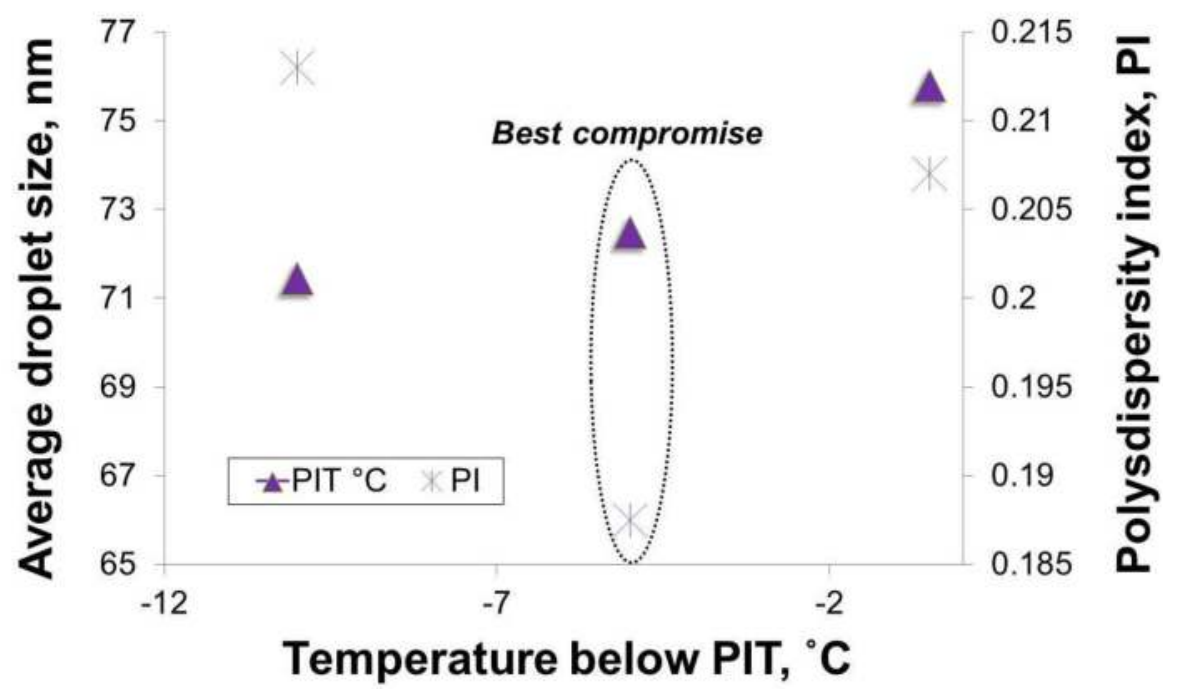

Fig. 9. Influence of polymerization temperature below PIT on final average particle size. Nanoemulsion formulation: $\phi_{\mathrm{oil}}=0.2, \mathrm{X}_{\mathrm{Brij} 700}=0.35$ and $5.2 \mathrm{wt} \%$ surfactant concentration. (Adapted from Galindo-Alvarez, et al., 2011)

Figure 10 illustrates the particle size obtained from two miniemulsion polymerization methods (low energy Near-PIT and ultrasound emulsification) and one coarse-emulsion polymerization (standard mechanical emulsification) with various surfactant to oil weight ratios and composition of surfactant mixture. In macroemulsion polymerization protocol, coarse-emulsion is agitated at $800 \mathrm{rpm}$ as in Near-PIT method, but the system is heated only until polymerization temperature and not $25{ }^{\circ} \mathrm{C}$ beyond as in Near-PIT. As expected, macroemulsion polymerization from coarse-emulsion gave the highest particle diameters and polydispersity indices which indicate a large and probably multimodal particle size distribution (see figures. 10 and 11). Thus, the viability of Near-PIT method to produce submicronic droplets as templates for miniemulsion polymerization, with slightly better efficiency than that found for ultrasonic emulsification method, has been confirmed. 


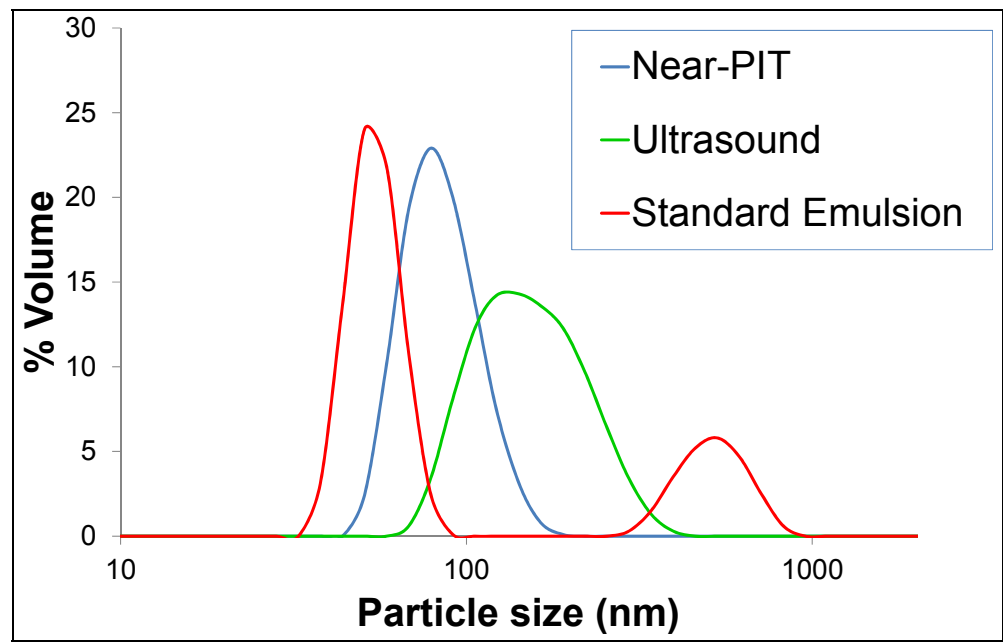

Fig. 10. Influence of emulsification process in particle size distribution, nanoemulsion formulation: ${ }^{\circ}$ oil $=0.35, \mathrm{X}_{\mathrm{Brij}} 700=0.4$ and $5.2 \mathrm{wt} \%$ surfactant concentration. (Adapted from Galindo-Alvarez, et al., 2011)

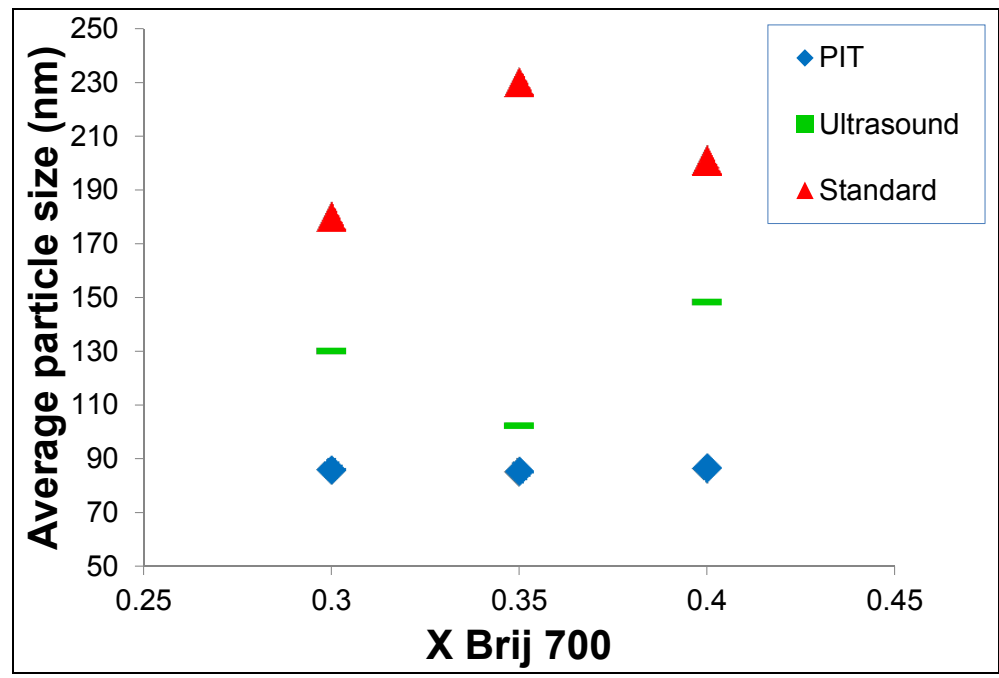

Fig. 11. Influence of emulsification process in average particle size, miniémulsion formulation: ${ }^{\circ}$ oil $=0.35$ and $5.2 \mathrm{wt} \%$ surfactant concentration. (Adapted from GalindoAlvarez, et al., 2011)

\section{Conclusion}

This chapter has showed the viability to produce polystyrene nanoparticles by two different types of low energy emulsification methods: EIP, emulsion inversion point and Near-PIT, near phase inversion temperature. 
The Emulsion Inversion Point technique was used on the water/Brij 98/styrene system to the formation of direct styrene-in-water nanoemulsions. After miniemulsion polymerization, particle sizes as low as $36 \mathrm{~nm}$ were obtained. These values are much lower than the one classically reached by high-energy emulsification methods. Thus Emulsion Inversion Point method is very attractive in industrial applications for nanoparticle synthesis, since the nano-emulsion formation does not require high concentration of surfactant, as in the case of microemulsion, or special high-shear equipments as in the case of most reported miniemulsion polymerization

The Phase Inversion Temperature concept as a tool to produce miniemulsion templates for miniemulsion polymerization is a promising methodology in polymerization field to obtain monodisperse and aggregate-free nanoparticle suspensions. In this review a low-energy emulsification method has been designed and showed to allow the preparation of polymeric particles smaller than usual ultrasound miniemulsification methods (about $75 \mathrm{~nm}$ ) in a water/Brij $78+$ Brij 700/styrene system. The operating conditions were adjusted so as to conciliate particle size distribution, colloidal stability and polymerization kinetics. Contrary to usual PIT methods, the Near-PIT procedure did not imply heating the samples at temperatures higher than PIT. Final particles had comparable characteristics to those obtained by traditional PIT methods. In addition, we showed the relevance of temperature control (PIT or PIT- $\Delta$ T) over nanoparticle size to obtain even slightly smaller particles than those obtained after ultrasound emulsification. Finally it is possible to vary the composition of the surfactant mixture, within a certain range, without strongly modifying nanoparticles final characteristics, but in way to control the thickness of the hydrophilic superficial layer.

\section{Acknowledgments}

This work has been funded by the BLAN-06-0174 ANR (National Research Agency) program. The authors thank Prof. Alain Durand, Dr. Marianna Rondon Gonzales, Mrs. Audrey Acrement, Mrs. Ioulia Habipi and Mr. David Boyd for their contributions to this study.

\section{References}

Anton, N., Benoit, J. P., \& Saulnier, P. (2008). Design and production of nanoparticles formulated from nano-emulsion templates-A review. Journal of Controlled Release, 128(3), 185-199.

Antón, R. E., Castillo, P., \& Salager, J.-L. (1986). Surfactant-oil-water systems near the affinity inversion part IV: emulsion inversion temperature. Journal of Dispersion Science and Technology 7(3), 319-329.

Antonietti, M., \& Landfester, K. (2002). Polyreactions in miniemulsions. Progress in Polymer Science (Oxford), 27(4), 689-757.

Asua, J. M. (2002). Miniemulsion polymerization. Progress in Polymer Science (Oxford), 27(7), 1283-1346.

Bouanani, F., Bendedouch, D., Hemery, P., \& Bounaceur, B. (2008). Encapsulation of montmorillonite in nanoparticles by miniemulsion polymerization. Colloids and Surfaces A: Physicochemical and Engineering Aspects, 317(1-3), 751-755. 
Calderó, G., García-Celma, M. J., \& Solans, C. (2011). Formation of polymeric nanoemulsions by a low-energy method and their use for nanoparticle preparation. Journal of Colloid And Interface Science, 353(2), 406-411.

Forgiarini, A., Esquena, J., Gonzalez, C., \& Solans, C. (2001). Formation of nano-emulsions by low-energy emulsification methods at constant temperature. Langmuir, 17 20762083.

Galindo-Alvarez, J., Boyd, D., Marchal, P., Tribet, C., Perrin, P., Marie-Bégué, E., et al. (2011). Miniemulsion polymerization templates: A systematic comparison between low energy emulsification (Near-PIT) and ultrasound emulsification methods. Colloids and Surfaces A: Physicochemical and Engineering Aspects, 374(1-3), 134-141.

Izquierdo, P., Feng, J., Esquena, J., Tadros, T. F., Dederen, J. C., Garcia, M. J., et al. (2005). The influence of surfactant mixing ratio on nano-emulsion formation by the pit method. Journal of Colloid And Interface Science, 285(1), 388-394.

Jahanzad, F., Chauhan, G., Mustafa, S., Saha, B., Sajjadi, S., \& Brooks, B. W. (2007). Composite Polymer Nanoparticles via Transitional Phase Inversion Emulsification and Polymerisation. Macromolecular Symposia, 259(1), 145-150.

Kabal'nov, A. S., Pertzov, A. V., \& Shchukin, E. D. (1987). Ostwald ripening in twocomponent disperse phase systems: Application to emulsion stability. Colloids and Surfaces, 24(1), 19-32.

Lamaallam, S., Bataller, H., Dicharry, C., \& Lachaise, J. (2005). Formation and stability of miniemulsions produced by dispersion of water/oil/surfactants concentrates in a large amount of water. Colloids and Surfaces A: Physicochemical and Engineering Aspects, 270-271(1-3), 44-51.

Maestro, A., Solè, I., González, C., Solans, C., \& Gutiérrez, J. M. (2008). Influence of the phase behavior on the properties of ionic nanoemulsions prepared by the phase inversion composition method. Journal of Colloid And Interface Science, 327(2), 433439.

Marie, E., Rotureau, E., Dellacherie, E., \& Durand, A. (2007). From polymeric surfactants to colloidal systems. 4. Neutral and anionic amphiphilic polysaccharides for miniemulsion stabilization and polymerization. Colloids and Surfaces A: Physicochemical and Engineering Aspects, 308(1-3), 25-32.

Mason, T. G., Graves, S. M., Wilking, J. N., \& Lin, M. Y. (2006). Extreme emulsification: Formation and structure of nanoemulsions. Condensed Matter Physics, 9(1), 193-199.

Mohlin, K., Holmberg, K., Esquena, J., \& Solans, C. (2003). Study of low energy emulsification of alkyl ketene dimer related to the phase behavior of the system. Colloids and Surfaces A: Physicochemical and Engineering Aspects, 218(1-3), 189-200.

Pey, C. M., Maestro, A., Solé, I., González, C., Solans, C., \& Gutiérrez, J. M. (2006). Optimization of nano-emulsions prepared by low-energy emulsification methods at constant temperature using a factorial design study. Colloids and Surfaces A: Physicochemical and Engineering Aspects, 288(1-3), 144-150.

Porras, M., Solans, C., González, C., \& Gutiérrez, J. M. (2008). Properties of water-in-oil (W/O) nano-emulsions prepared by a low-energy emulsification method. Colloids and Surfaces A: Physicochemical and Engineering Aspects, 324(1-3), 181-188.

Rang, M. J., \& Miller, C. A. (1999). Spontaneous emulsification of oils containing hydrocarbon, nonionic surfactant, and oleyl alcohol. Journal of Colloid And Interface Science, 209(1), 179-192. 
Sadtler, V., Rondon-Gonzalez, M., Acrement, A., Choplin, L., \& Marie, E. (2010). PEOCovered Nanoparticles by Emulsion Inversion Point (EIP) Method. Macromolecular Rapid Communications, 31(11), 998-1002.

Sadurní, N., Solans, C., Azemar, N., \& García-Celma, M. J. (2005). Studies on the formation of $\mathrm{O} / \mathrm{W}$ nano-emulsions, by low-energy emulsification methods, suitable for pharmaceutical applications. European Journal of Pharmaceutical Sciences, 26(5), 438445.

Salager, J.-L. (1988). 2 Phase transformation and emulsion inversion on the basis of catastrophe theory. In P. Becher (Ed.), Encyclopedia of Emulsion Technology: Basic Theory, Measurement, Applications (Vol. 3, pp. 79 - 134). New York - Basel: Marcel Dekker.

Salager, J.-L. (2000a). Emulsion properties and related know-how to attain them. In F. Nielloud \& G. Marti-Mestres (Eds.), Pharmaceutical Emulsions and Suspensions (pp. 73 - 125). New York: Marcel Dekker.

Salager, J.-L. (2000b). Formulation concepts for the emulsion maker. In F. Nielloud \& G. Marti-Mestres (Eds.), Pharmaceutical Emulsions and Suspensions (pp. 19 - 72). New York: Marcel Dekker.

Salager, J.-L., Miñana-Perez, M., M. Pérez-Sánchez, Ramirez-Gouveia, M., \& Rojas, C. I. (1983). Surfactant-oil-water systems near the affinity inversion part III: the two kinds of emulsion inversion. Journal of Dispersion Science and Technology 4(3), 313 329.

Salager, J.-L., Perez-Sanchez, M., \& Garcia, Y. (1996). Physicochemical parameters influencing the emulsion drop size. Colloid and Polymer Science, 274(1), 81-84.

Schork, F. J., Luo, Y., Smulders, W., Russum, J. P., Butté, A., \& Fontenot, K. (2005). Miniemulsion Polymerization Polymer Particles (pp. 129-255).

Shinoda, K., \& Arai, H. (1964). The correlation between phase inversion temperature in emulsion and cloud point in solution of nonionic emulsifier. Journal of Physical Chemistry, 68(12), 3485-3490.

Shinoda, K., \& Arai, H. (1967). The effect of phase volume on the phase inversion temperature of emulsions stabilized with nonionic surfactants. Journal of Colloid And Interface Science, 25(3), 429-431.

Shinoda, K., \& Saito, H. (1968). The effect of temperature on the phase equilibria and the types of dispersions of the ternary system composed of water, cyclohexane, and nonionic surfactant. Journal of Colloid and Interface Science, 26(1), 70-74.

Solans, C., Esquena, J., Forgiarini, A. M., Uson, N., Morales, D., Izquierdo, P., et al. (2002). Nanoemulsions: Formation and Properties. In K. L. Mittal \& D. O. Shah (Eds.), Surfactants in Solution: Fundamentals and Applications (pp. 525). New York: Marcel Dekker.

Solans, C., Izquierdo, P., Nolla, J., Azemar, N., \& Garcia-Celma, M. J. (2005). Nanoemulsions. Current Opinion in Colloid \& Interface Science, 10(3-4), 102-110.

Solè, I., Maestro, A., González, C., Solans, C., \& Gutiérrez, J. M. (2006). Optimization of nano-emulsion preparation by low-energy methods in an ionic surfactant system. Langmuir, 22(20), 8326-8332.

Solè, I., Pey, C. M., Maestro, A., González, C., Porras, M., Solans, C., et al. (2010). Nanoemulsions prepared by the phase inversion composition method: Preparation variables and scale up. Journal of Colloid And Interface Science, 344(2), 417-423. 
Spernath, L., \& Magdassi, S. (2007). A new method for preparation of poly-lauryl acrylate nanoparticles from nanoemulsions obtained by the phase inversion temperature process. Polymers for Advanced Technologies, 18(9), 705-711.

Spernath, L., \& Magdassi, S. (2010). Formation of silica nanocapsules from nanoemulsions obtained by the phase inversion temperature method. Micro and Nano Letters, 5(1), 28-36.

Spernath, L., Regev, O., Levi-Kalisman, Y., \& Magdassi, S. (2009). Phase transitions in O/W lauryl acrylate emulsions during phase inversion, studied by light microscopy and cryo-TEM. Colloids and Surfaces A: Physicochemical and Engineering Aspects, 332(1), 19-25.

Tadros, T., Izquierdo, P., Esquena, J., \& Solans, C. (2004). Formation and stability of nanoemulsions. Advances in Colloid and Interface Science, 108-109, 303-318. 


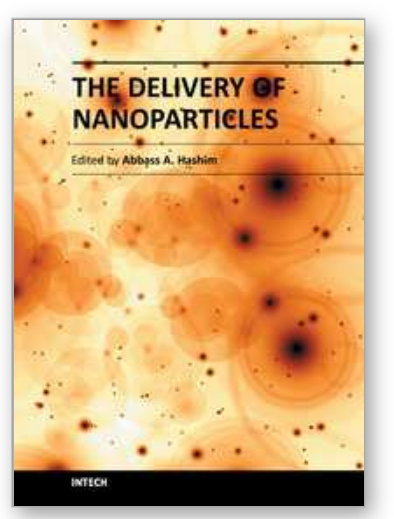

\author{
The Delivery of Nanoparticles \\ Edited by Dr. Abbass A. Hashim
}

ISBN 978-953-51-0615-9

Hard cover, 540 pages

Publisher InTech

Published online 16, May, 2012

Published in print edition May, 2012

Nanoparticle is a general challenge for today's technology and the near future observations of science. Nanoparticles cover mostly all types of sciences and manufacturing technologies. The properties of this particle are flying over today scientific barriers and have passed the limitations of conventional sciences. This is the reason why nanoparticles have been evaluated for the use in many fields. InTech publisher and the contributing authors of this book in nanoparticles are all overconfident to invite all scientists to read this new book. The book's potential was held until it was approached by the art of exploring the most advanced research in the field of nano-scale particles, preparation techniques and the way of reaching their destination. 25 reputable chapters were framed in this book and there were alienated into four altered sections; Toxic Nanoparticles, Drug Nanoparticles, Biological Activities and Nano-Technology.

\title{
How to reference
}

In order to correctly reference this scholarly work, feel free to copy and paste the following:

Veronique Sadtler, Johanna M. Galindo-Alvarez and Emmanuelle Marie -Bégué (2012). Low Energy Emulsification Methods for Nanoparticles Synthesis, The Delivery of Nanoparticles, Dr. Abbass A. Hashim (Ed.), ISBN: 978-953-51-0615-9, InTech, Available from: http://www.intechopen.com/books/the-delivery-ofnanoparticles/low-energy-emulsification-methods-for-nanoparticles-synthesis

\section{INTECH}

open science | open minds

\author{
InTech Europe \\ University Campus STeP Ri \\ Slavka Krautzeka 83/A \\ 51000 Rijeka, Croatia \\ Phone: +385 (51) 770447 \\ Fax: +385 (51) 686166 \\ www.intechopen.com
}

\author{
InTech China \\ Unit 405, Office Block, Hotel Equatorial Shanghai \\ No.65, Yan An Road (West), Shanghai, 200040, China \\ 中国上海市延安西路65号上海国际贵都大饭店办公楼 405 单元 \\ Phone: +86-21-62489820 \\ Fax: +86-21-62489821
}


(C) 2012 The Author(s). Licensee IntechOpen. This is an open access article distributed under the terms of the Creative Commons Attribution 3.0 License, which permits unrestricted use, distribution, and reproduction in any medium, provided the original work is properly cited. 